\title{
Introduction
}

\section{Editorial: Considering Transient Instantiators}

\author{
Jed T. Elison ${ }^{1,2}$ \\ ${ }^{1}$ Institute of Child Development, University of Minnesota, Minneapolis, MN, USA and ${ }^{2}$ Department of Pediatrics, University of Minnesota, Minneapolis, MN, USA
}

\begin{abstract}
"Until we answer that question about heterogeneity, which presupposes answering the specific question of specific etiology, especially how "specificity" is to be construed for a polygenic theory, we do not know whether two labels [or more] are needed, let alone to which patients they should be applied." Meehl (1972) (p. 382).
\end{abstract}

Among several key themes, the authors of the contributions to this special section of Development and Psychopathology were tasked to address heterogeneity. Core principles of developmental psychopathology such as equifinality and multifinality (Cicchetti \& Rogosch, 1996) map onto each etiological and phenotypic heterogeneity, respectively. Here, we would like to highlight an additional source of variability that exists between causal pathophysiological instantiators and a phenotypic endpoint, and the conceptual consequences thereof. Temporal heterogeneity, a term borrowed from ecological theory (Dutilleul, 2011; Menge \& Sutherland, 1976) and not unrelated to the concept of chronogeneity (Georgiades, Bishop, \& Frazier, 2017), considered in the context of neurodevelopmental disorders, captures observed variability in developmental timing as it relates to pathogenesis. Temporal heterogeneity may represent a specific feature of phenotypic heterogeneity. It may also capture evolving patterns of environmental demands that temporally coincide and interact with changes in the developing organism (Lehrman, 1953). By simply invoking the concept, however, we hope to illustrate a developmental frame, captured by many of the contributions in this special section, but less well appreciated in the broader autism field. Implicit to this framework is an expectation that it is unlikely that a given phenotypic profile will remain stable and persistent, or static over time (Karmiloff-Smith, 1998).

As conceptualized for psychiatric disorders (Insel \& Cuthbert, 2015) and autism specifically (Geschwind \& State, 2015; Loth, Murphy, \& Spooren, 2016), the overarching precision medicine framework explicitly attempts to model etiological and phenotypic heterogeneity in order to deliver the right intervention, at the right dose, to the right person, at the right time (Collins \& Varmus, 2015). Make no mistakes, without an adequate conceptual framework, the right time criterion will remain a thorn in our proverbial side. Following an empirical publication elucidating the phenomenon of temporal heterogeneity as conceptualized herein (Anticevic et al., 2015), albeit with cross-sectional data,

Author for Correspondence: Jed T. Elison, 51 East River Parkway, Minneapolis, MN 55455; E-mail: jtelison@umn.edu

Cite this article: Elison JT (2020). Editorial: Considering Transient Instantiators. Development and Psychopathology 32, 1173-1174. https://doi.org/10.1017/ S0954579420001807
Krystal and Anticevic (2015, p. 738 ) state "it may be timely to draw attention to one potential source of heterogeneity within and across patients - the dynamic time-dependent neurobiological evolution of schizophrenia across its course of illness." While the search for biomarkers that could serve as treatment targets for neurodevelopmental disorders is a noble enterprise, the assumption that biomarkers are stable and persistent across time and illness phase requires empirical data to refute or fail to refute said assumption/hypothesis.

Indeed, for a special class of biomarkers, endophenotypes (Gottesman \& Gould, 2003; Gottesman \& Shields, 1972), inherent to the definition is that the marker be state independent, or in other words, that it be present whether someone with schizophrenia is in a prodromal state, an actively ill state, or in a state of symptom remission. Quasi-developmentally oriented conceptualizations of the endophenotype also posit that it should be operable prior to any symptom manifestation (Iacono \& Malone, 2011; Lenzenweger, 2013; Skuse, 2001). Additional poignant commentary presaged the observation from Krystal and Anticevic (2015) referenced above, "[i]deally, to be an appropriate endophenotype, a risk factor should be correlated with disease and/or disease severity but it should be clearly established that this correlation is not a consequence of either medication or degeneration due to disease progression" (Almasy \& Blangero, 2001, p. 42). "Disease [or rather disorder] progression" is integral to neurodevelopmental disorders - and anchors our conceptualization of temporal heterogeneity. Therefore, unless one clings to the conceptualization of neurodevelopmental or psychiatric phenotypes, and consequently the biomarkers that underlie symptom dimensions or symptom profiles, as stable and persistent, the search for endophenotypes appears less Herculean and more Sisyphean.

If we afford some logical coherence to the concept of temporal heterogeneity, an amendment to the state independence criterion may be warranted. Namely, we propose that an endophenotype must be observed prior to the manifestation of the constellation of features that define a given condition/disorder. Importantly, this marker may or may not persist through the developmental course of the condition/disorder. If it does persist, it may be subsequently obfuscated by disease/disorder progression. Indeed, the likelihood of what Meehl (1972) referred to as a potentiator and what we refer to as an instantiator not being obscured by disease/disorder progression is slim. Therefore, the search for transient instantiators that fulfill the expanded list of endophenotype criteria sans the traditional state-independence criterion may yield promising avenues for exploration. This work will not be 
easy. However, work in this special section showcases the type of developmental thinking that will push us in the right direction.

Acknowledgments. The funders had no role in the writing of this report. JTE expresses his deep appreciation to Dante Cicchetti for his support and mentorship. The ideas herein germinated and were nourished by conversations with and the mentorship of Joe Piven and Irv Gottesman.

Funding Statement. JTE was supported by NIMH R01 MH104324.

Conflicts of Interest. None

\section{References}

Almasy, L., \& Blangero, J. (2001). Endophenotypes as quantitative risk factors for psychiatric disease: Rationale and study design. American Journal of Medical Genetics (Neuropsychiatric Genetics), 105, 42-44.

Anticevic, A., Corlett, P. R., Cole, M. W., Savic, A., Gancsos, M., Tang, Y., et al. (2015). N-methyl-D-aspartate receptor antagonist effects on prefrontal cortical connectivity better model early than chronic schizophrenia. Biological Psychiatry, 77, 569-580.

Cicchetti, D., \& Rogosch, F. (1996). Editorial: Equifinality and multifinality in developmental psychopathology. Development and Psychopathology, 8, 597-600.

Collins, F. S., \& Varmus, H. (2015). A new initiative on precision medicine. New England Journal of Medicine, 372, 793-795.

Dutilleul, P. R. L. (2011). Spatio-temporal heterogeneity: Concepts and analyses. New York, NY: Cambridge University Press.

Georgiades, S., Bishop, S. L., \& Frazier, T. (2017). Editorial perspective: Longitudinal research in autism - introducing the concept of 'chronogeneity'. Journal of Child Psychology and Psychiatry, 58, 634-636.
Geschwind, D. H., \& State, M. W. (2015). Gene hunting in autism spectrum disorder: On the path to precision medicine. Lancet Neurology, 14, 1109-1120. Gottesman, I. I., \& Gould, T. D. (2003). The endophenotype concept in psychiatry: Etymology and strategic intentions. American Journal of Psychiatry, 160, 636-645.

Gottesman, I. I., \& Shields, J. (1972). Schizophrenia and genetics: A twin study vantage point. New York: Academic Press.

Iacono, W. G., \& Malone, S. M. (2011). Developmental endophenotypes: Indexing genetic risk for substance abuse with the P300 brain event-related potential. Child Development Perspectives, 5, 239-247.

Insel, T. R., \& Cuthbert, B. N. (2015). Brain disorders? Precisely. Science, 348, 499-500.

Karmiloff-Smith, A. (1998). Development itself is the key to understanding developmental disorders. Trends in Cognitive Sciences, 2, 389-398.

Krystal, J. H., \& Anticevic, A. (2015). Commentary: Toward illness phasespecific pharmacology for schizophrenia. Biological Psychiatry, 78, 738-740.

Lehrman, D. S. (1953). A critique of Konrad Lorenz's theory of instinctive behavior. Quarterly Review of Biology, 28, 337-363.

Lenzenweger, M. F. (2013). Thinking clearly about the endophenotypeintermediate phenotype-biomarker distinctions in developmental psychopathology research. Development \& Psychopathology, 25, 1347-1357.

Loth, E., Murphy, D. G., \& Spooren, W. (2016). Defining precision medicine approaches to autism spectrum disorders: Concepts and challenges. Frontiers of Psychiatry, 7, 188.

Meehl, P. E. (1972). A critical afterward. In I. I. Gottesman \& J. Shields (Eds.), Schizophrenia and genetics: A twin study vantage point (pp. 131-189). New York: Academic Press.

Menge, B. A., \& Sutherland, J. P. (1976). Species diversity gradients: Synthesis of the roles of predation, competition, and temporal heterogeneity. The American Naturalist, 110, 351-369.

Skuse, D. H. (2001). Endophenotypes and child psychiatry. British Journal of Psychiatry, 178, 395-396. 